\title{
Aqueous extract of betel nut-induced adducts on pMTa4 DNA acquires stability in the presence of $\mathrm{Na}^{+}$and $\mathrm{K}^{+}$ions
}

\author{
CHAITALI BHATTACHARJEE and RAJESHWAR NATH SHARAN \\ Radiation and Molecular Biology Unit, Department of Biochemistry, North Eastern Hill University, Shillong 793022, India
}

Received December 27, 2007; Accepted February 11, 2008

\begin{abstract}
Betel nut (BN), a natural carcinogen to humans, is used as a masticator across the globe by a large segment of the human population. The primary carcinogens of $\mathrm{BN}$ are alkaloids, in particular arecoline. Upon nitrosation, arecoline can potentially interact with DNA, forming adducts and initiating carcinogenesis. Though considerable evidence exists in support of the carcinogenicity of $\mathrm{BN}$, the molecular mechanism of its induction of carcinogenesis is unknown. This investigation was undertaken to directly demonstrate adduct formation on DNA and to study its characteristics, such as its frequency of formation and stability. A plasmid DNA construct, pMTa4, was chosen to determine the stoichiometry and dynamics of adduct formation. This construct was exposed to aqueous extract of betel nut (AEBN) in vitro and in vivo and analyzed. Spectrophotometric analysis revealed a significant red shift in the pMTa4 DNA spectrum. The gel electrophoretic mobility of pMTa4 DNA was also retarded in an AEBN dose- and exposure time-dependent manner, indicating BN-specific adduct formation on the DNA. These results conclusively demonstrate that adducts are formed on DNA by $\mathrm{BN}$ extract, and suggest that one AEBN-induced adduct was formed every 3 NT on pMTa4 DNA under the experimental conditions. Trace amounts of monovalent cations, such as $\mathrm{Na}^{+}$or $\mathrm{K}^{+}$ion $(\geq 0.5 \mathrm{mmol})$, conferred stability to the adducts on DNA, which were otherwise unstable beyond $24 \mathrm{~h}$.
\end{abstract}

\section{Introduction}

Humans are constantly exposed to carcinogenic agents that interact with DNA directly or indirectly, thus inducing damage which can lead to a variety of diseases, including cancer. Betel nut (BN; Areca catechu L.) is one such agent, and is implicated in the induction of a wide range of cancers, including cancers of the oropharyngeal tract and hepatocarcinomas. Despite its

Correspondence to: Professor R.N. Sharan, Department of Biochemistry, North-Eastern Hill University, Umshing, Shillong 793022, India

E-mail: rnsharan@nehu.ac.in

Key words: aqueous extract of betel nut, pMTa4 DNA, betel nut specific adduct, gel mobility shift, stability, monovalent cations reported association with carcinogenicity, it is estimated that $\mathrm{BN}$ in various forms is widely used as a masticator by over 600 million people across the globe (reviewed in refs. 1-4). In chronic chewers, it is especially implicated in oral leukoplakia and oral submucous fibrosis (OSF), and is believed to be one of the major reasons for the high incidence of oral malignancy found in Asian populations (5). Detailed physicochemical investigations have established that water-soluble alkaloids, besides some polyphenols and tannins, are the main carcinogenic component of $\mathrm{BN}(1,3)$. BN alkaloid consists predominantly of arecoline and arecaidine, as well as small amounts of guvacine and guvacoline, among others $(1,3,4)$. Considerable evidence suggests that arecoline, upon nitrosation, produces at least four types of betel nut-specific nitrosamines (BSNAs) $(1,3)$. These compounds have been isolated, purified and characterized. Being electrophilic, they have a high affinity for DNA $(1,3)$ and consequently interact with it readily. This can produce DNA adducts (6) that potentially initiate mutagenesis, leading to carcinogenesis (7).

Arecoline and various BN extracts - in particular aqueous extract of betel nut (AEBN) - have been shown to be cytostatic and cytotoxic to the human hep 2 cell line (8), as well as being tumorigenic (9). They induce dysregulated cell cycle controls in OSF and interfere with the glutathione metabolism (10). It has been demonstrated that the metabolic activation of arecoline occurrs via nitrosation in the hep2 cell line (8). This exposure induces strand breaks in DNA and an enhanced cell cycle (11), and prompts different types of chromosomal aberrations, including sister chromatid exchanges (10) and unscheduled DNA synthesis (UDS) (12). However, BN has not been reported to induce cellular apoptosis (13). A 2-fold induction of $c$-fos and $c$-jun protooncogene products as well as elevated $c$-Myc levels in JB6 cell lines upon exposure to betel quid suggests the induction of cellular transformation (13). These observations strongly indicate that components of BN, especially alkaloids and their activated derivatives, might form adducts on DNA. Indeed, the presence of BSNA adducts following exposure to $\mathrm{BN}$ or betel quid has been chemically demonstrated. Quantification of induced adducts by fluorescence-detected HPLC, gas chromatography or other biophysical methods has shown a high degree of correlation with cellular transformation (14). Thus, the involvement of adducts in BN-induced human carcinogenesis is clear. Nonetheless, to the best of our knowledge not much is known about the molecular characteristics of the DNA adducts or their consequences (3). In particular, the kinetics of BN-specific adduct formation on DNA and factors affecting their stability are totally unknown. This paper reports 
on an investigation of the molecular characterization and kinetics of AEBN-induced in vitro and in vivo formation of DNA adducts.

Our chosen model was E. coli, as this simple organism has been used with great success in past studies to determine the molecular characteristics of adduct formation. E. coli is also preferred because it often harbors or can be made to harbor plasmid DNA, which is a convenient tool for this type of study (15). Our laboratory has designed a plasmid DNA construct, pMTa4 (6173 bp), which has been successfully used to reveal the DNA damage inflicted by free radicals (16) or by different qualities of radiation $(17,18)$. Other groups have used similar approaches (19). The plasmid pMTa4 was used in this study as well. For in vitro investigation, isolated plasmid DNA was exposed to AEBN in an Eppendorf tube and analyzed. For in vivo studies, $\mathrm{pMTa} 4$ was first transformed into a wild-type $E$. coli strain, AB1157. The transformed E. coli cells harboring pMTa4 were then exposed to AEBN under physiological conditions. Following AEBN exposure, pMTa4 was isolated from the $E$. coli cells and analyzed.

\section{Materials and methods}

Chemicals. All chemicals used were of the highest purity grade. Agarose, $\lambda$ DNA HindIII digest (Genei, India), ampicillin (Duchefa, The Netherlands), ethidium bromide (EB; Merck, Germany), Tris-base (Boehringer Mannheim GmbH, Germany), ethylenediaminetetracetic acid (EDTA) and sodium dodecyl sulphate (SDS; Sigma Chemical Co., USA), KCl, $\mathrm{HCl}$, acetic acid and sodium hydroxide (Qualigens, India), sodium acetate (SRL, India), $\mathrm{NaCl}$, Luria Bertani (LB) broth and LB-agar (Himedia, India), and ethanol (Bengal Chemicals, India) were used.

Preparation of aqueous extract of betel nut. AEBN was prepared as previously described (20). Briefly, raw and wet varieties of $\mathrm{BN}$, obtained from a local market, were dehusked, crushed to a coarse powder and soaked overnight in sterile water at room temperature. The extract was then filtered through Whatman No. 1 filter paper and lyophilized to powder. The resulting AEBN was dissolved in sterile water at appropriate concentrations $(50,100,150,200$ and $250 \mu \mathrm{g})$ for the experiment.

Plasmid pMTa4 and its isolation. The previously described pMTa4 plasmid (15-18) was isolated by standard alkaline methods from an overnight mid-log culture of $E$. coli in LB broth with $100 \mu \mathrm{g} / \mu \mathrm{l}$ Ampicillin at $37^{\circ} \mathrm{C}$. The inoculum for the culture was a single colony picked up from an Ampicillin ${ }^{+}$LBagar plate of a wild (AB1157) K12 strain of E. coli harboring the plasmid. The pMTa4 isolate was air dried, dissolved in sterile water and refrigerated until use.

Transformation of pMTa4 in E. coli. Transformation was performed as recently described (18). In brief, $\approx 75 \mu 1$ (300 ng DNA) of pMTa4 was added to $200 \mu 1$ of freshly prepared competent cells in a pre-cooled tube, gently mixed and kept on ice for $20 \mathrm{~min}$. The tube was then incubated in sequence at $42^{\circ} \mathrm{C}$ for $30 \mathrm{sec}$ and on ice for $180 \mathrm{sec}$. LB medium (500 $\mu 1$, pre-warmed to $37^{\circ} \mathrm{C}$ ) was added to the tube, gently mixed, and incubated at $37^{\circ} \mathrm{C}$ for $60 \mathrm{~min}$. The contents $(200 \mu \mathrm{l})$ were then plated on LB-agar plates (with $100 \mu \mathrm{g} / \mathrm{ml}$ Ampicillin) at $10^{-3}$ and $10^{-5}$ dilutions, then incubated overnight at $37^{\circ} \mathrm{C}$. An LB-agar plate without ampicillin was used as the control.

Agarose gel electrophoresis. Electrophoresis was performed on a $1 \%$ agarose gel using TAE $(40 \mathrm{mM}$ Tris-acetate, $1 \mathrm{mM}$ EDTA) running buffer at $100 \mathrm{~V}$ (constant) for $60 \mathrm{~min}$ as previously described $(16,18)$. The gel was stained with EB $(0.3 \mu \mathrm{g} / \mathrm{ml})$ for $15 \mathrm{~min}$, then destained in water for $30 \mathrm{~min}$ on a rocking plate. The EB-intercalated DNA was visualized on a UV transilluminator (Bio-Rad).

Spectrophotometric analysis of pMTa4 DNA following incubation with $A E B N$ in vitro. pMTa4 isolates $(2 \mu 1$ containing $4 \mu \mathrm{g}$ DNA) were incubated at $37^{\circ} \mathrm{C}$ without or with varying concentrations of AEBN (50,100, 150, 200 and $250 \mu \mathrm{g})$ for different time intervals $(0,15$ and $30 \mathrm{~min})$. The mixtures were diluted to $1 \mathrm{ml}$ with sterile water and the absorbance spectra were recorded over a range of 235-320 nm using a spectrophotometer (Systronics, UV-Vis Spectrophotometer 119).

AEBN-induced mobility shift of pMTa4 as a function of the concentration of $A E B N$ in vitro. pMTa4 isolates ( $2 \mu 1$ containing $4 \mu \mathrm{g}$ DNA) were incubated at $37^{\circ} \mathrm{C}$ for $30 \mathrm{~min}$ without or with varying concentrations of $\operatorname{AEBN}(50,100$, 150,200 and $250 \mu \mathrm{g}$ ). Following exposure, the samples were subjected to agarose gel electrophoresis.

AEBN-induced mobility shift of pMTa4 as a function of the time of incubation in vitro. pMTa4 isolates $(2 \mu 1$ containing $4 \mu \mathrm{g}$ DNA) were incubated with $250 \mu \mathrm{g}$ of AEBN at $37^{\circ} \mathrm{C}$ for different time periods $(0,5,10,15,20,25,30,35,40$ and $45 \mathrm{~min})$, then subjected to agarose gel electrophoresis.

Stability of AEBN-DNA adduct in vivo. AEBN (2000 $\mu \mathrm{g} \mathrm{ml})$ was added to a flask containing LB medium and $100 \mu \mathrm{g} / \mu \mathrm{l}$ ampicillin. It was inoculated with a single colony of $E$. coli from an ampicillin ${ }^{+}$-agar plate. The control was normal medium without AEBN. The cultures were grown overnight at $37^{\circ} \mathrm{C}$. Plasmid was then isolated from the cultures. Equimolar aliquots of pMTa4 isolate were dissolved in $30 \mu 1$ of sterile water and incubated at room temperature for increasing time intervals up to $24 \mathrm{~h}$. Samples were taken out every two hours for evaluation by agarose gel electrophoresis.

Effects of $\mathrm{pH}$ and the $\mathrm{Na}^{+}$and $\mathrm{K}^{+}$ions on the stability of $A E B N$-induced adducts. In order to study the effects of $\mathrm{pH}$ and monovalent cations on the stability of AEBN-induced pMTa4 adducts, equimolar amounts of air-dried pMTa4 DNA (isolates) were taken in different tubes. To study the effects of $\mathrm{pH}$ on the stability, the pMTa4 isolates were dissolved separately in $30 \mu 1$ of $1 \mathrm{M}$ Tris- $\mathrm{Cl}$ buffers at $\mathrm{pH} 5.0,6.0,7.0$ or 8.0. The control pMTa4 DNA was dissolved in sterile water. The tubes were incubated at room temperature for $24 \mathrm{~h}$, then subjected to agarose gel electrophoresis. To study the effects of monovalent cations (e.g., $\mathrm{Na}^{+}$and $\mathrm{K}^{+}$) on stability, the pMTa4 isolates were dissolved separately in $30 \mu 1$ sterile water containing different concentrations of either $\mathrm{KCl}(0.5,5,50$ and $500 \mathrm{mmol})$ or $\mathrm{NaCl}(0.5,5,50$ and $500 \mathrm{mmol})$. pMTa4 

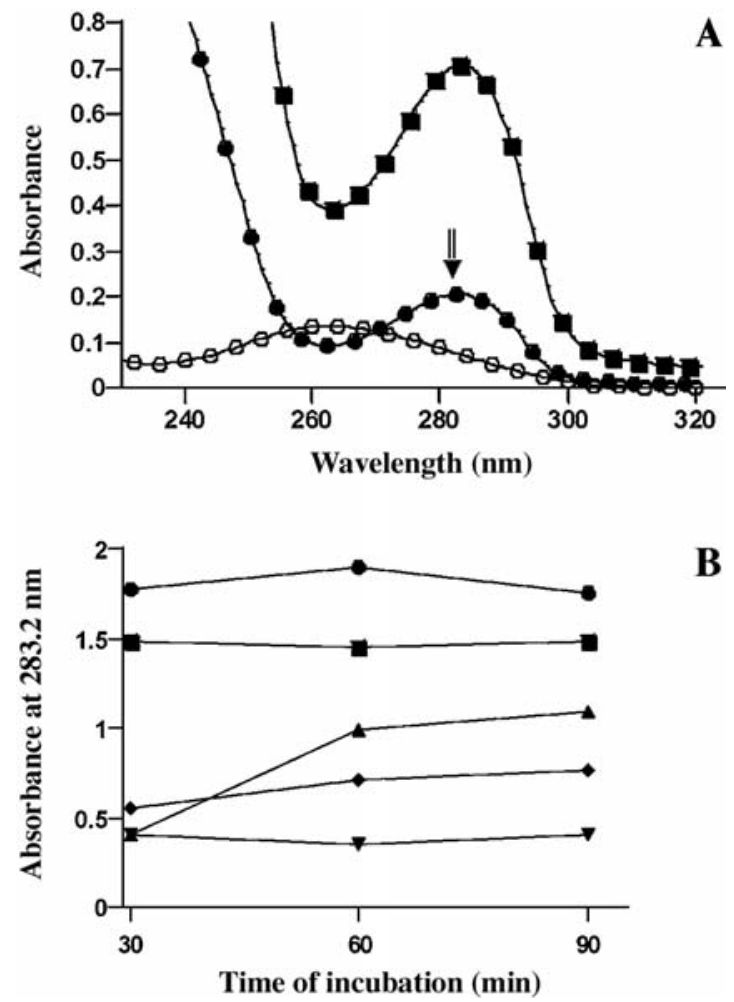

B

Figure 1. Spectrophotometric analyses of pMTa4 and AEBN interaction in vitro. (A) Absorbance spectra of pMTa4 DNA ( $)$, AEBN (-) and pMTa4 exposed to $250 \mu \mathrm{g} \mathrm{AEBN}$ for $30 \mathrm{~min}$ at $37^{\circ} \mathrm{C}(\bullet)$. (B) Absorbance maxima $(283.20 \mathrm{~nm})$ of pMTa4 exposed to $50 \mu \mathrm{g}(\boldsymbol{\nabla}), 100 \mu \mathrm{g}(\bullet), 150 \mu \mathrm{g}(\mathbf{\Delta}), 200 \mu \mathrm{g}$ (๘) and $250 \mu \mathrm{g}(\bullet) \mathrm{AEBN}$ for increasing time periods at $37^{\circ} \mathrm{C}$.

DNA dissolved in sterile water served as the control. The samples were incubated at room temperature for $24 \mathrm{~h}$, then subjected to agarose gel electrophoresis.

$A E B N$-induced increase in the molecular size of the linear form of pMTa4. The plasmid (pMTa4) was linearized using a restriction endonuclease, $\mathrm{NcoI}$, which has only one restriction site in the plasmid $(16,18)$. Aliquots $(2 \mu 1)$ of the linear (L) form of pMTa4 $(4 \mu \mathrm{g})$ were incubated without or with increasing concentrations of $\operatorname{AEBN}(50,100,150,200$ and $250 \mu \mathrm{g})$ at $37^{\circ} \mathrm{C}$ for $30 \mathrm{~min}$, then subjected to agarose gel electrophoresis.

Data capture, analysis and statistical evaluation. A minimum of five independent experiments were conducted for each investigation/point. The plasmid DNA bands on agarose gels were digitized (Kodak) immediately after destaining. The band intensities of different topological forms of pMTa4 were calculated as previously described $(16,18)$. From the digitized photographs, the mobility of the bands was calculated using gel documentation and analysis software (KDS1D; Kodak). The mean \pm SEM of all the data was calculated, and graphs were plotted using Kaleidagraph software. The student's t-test was applied for statistical evaluation. $\mathrm{p} \leq 0.01$ was considered significant.

\section{Results}

The method of plasmid isolation employed by the study routinely produced $\approx 40 \mu \mathrm{g}$ DNA from a culture of $9 \times 10^{9}$ E. coli cells. The plasmid isolate was dissolved in sterile water

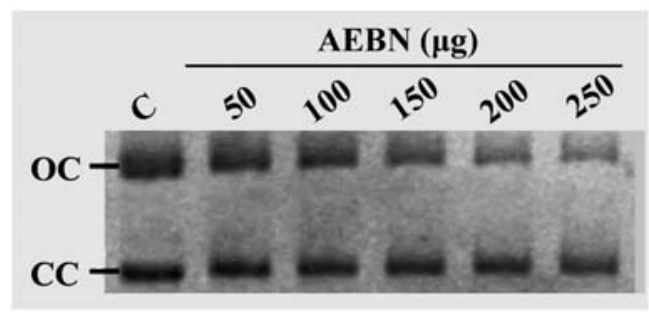

A

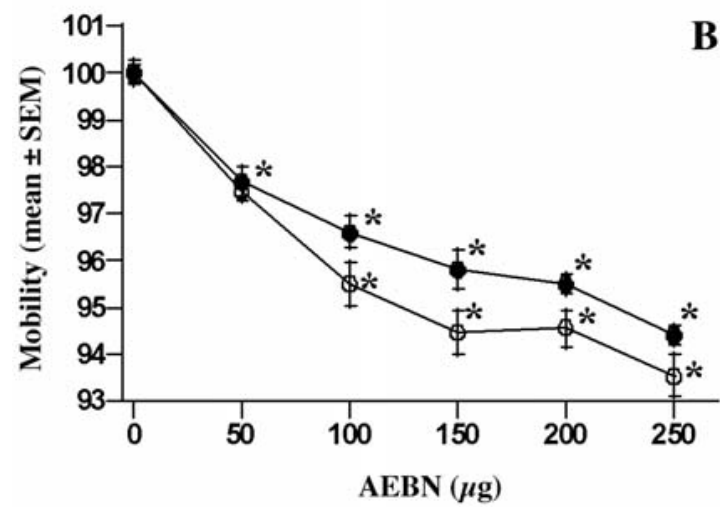

Figure 2. Effect of increasing doses of AEBN on the gel electrophoretic mobility of plasmid pMTa4 in vitro. (A) Retardation of the mobility of the $\mathrm{CC}$ and $\mathrm{OC}$ forms of pMTa4 with increasing doses of AEBN [lane 1 (C), unexposed pMTa4 (4 $\mu \mathrm{g})$; lanes 2-6, pMTa4 $(4 \mu \mathrm{g})$ exposed to 50, 100, 150, 200 and $250 \mu \mathrm{g}$ of $\mathrm{AEBN}$ respectively for $30 \mathrm{~min}$ at $37^{\circ} \mathrm{C}$ ]. (B) Percentage mobility changes for the $\mathrm{CC}(\bullet)$ and OC $(\circ)$ topological forms of pMTa4 as a function of the increasing dose of AEBN. Data (mean \pm SEM) were obtained from the electropherogram (A). *Statistically significant $(\mathrm{p} \leq 0.01)$ compared to the controls.

at a concentration of $2 \mu \mathrm{g} / \mu 1$. The purity of the isolate was high (a ratio of $260 / 280, \approx 1.9$ ), and the native conformation of the plasmids condensed, covalently closed circular (CC). However, during plasmid isolation some single strand breaks were inevitably induced on the $\mathrm{CC}$ form and resulted in the formation of an open circle (OC) or relaxed topological form of the plasmid $(16,18)$. The plasmid isolates used included about $60 \% \mathrm{CC}$ and $40 \%$ OC forms. The transformation frequency of pMTa4 plasmids into $E$. coli was moderate to high according to the protocol used.

$A E B N$-induced spectral red shift in vitro. Fig. 1A shows the typical absorbance spectra of pMTa4, AEBN and pMTa4 exposed to $250 \mu \mathrm{g}$ AEBN for $30 \mathrm{~min}$ at $37^{\circ} \mathrm{C}$. As expected, pMTa4 reached absorption maximum at $260.80 \mathrm{~nm}$, while AEBN peaked at $283.20 \mathrm{~nm}$. AEBN-exposed pMTa4 showed maximum absorption at $283.20 \mathrm{~nm}$ (arrow), recording a red spectral shift of $19.60 \mathrm{~nm}$ as compared to pMTa4. Fig. 1B plots the absorbance maxima $(283.20 \mathrm{~nm})$ of pMTa4 exposed to increasing doses of AEBN for different time periods at $37^{\circ} \mathrm{C}$. A time-dependent increase in absorbance was only observed for a dose of $150 \mu \mathrm{g}$ AEBN.

AEBN concentration-dependent gel mobility shift of pMTa4 DNA in vitro. The gel in Fig. 2A shows CC and OC topological forms of unexposed control pMTa4 (lane 1) and pMTa4 exposed to increasing doses of AEBN (lanes 2-6). Retardation of the mobility of both the $\mathrm{CC}$ and $\mathrm{OC}$ forms is noticeable. The plot of percent mobility shift as compared to the mobility of the $\mathrm{CC}$ and $\mathrm{OC}$ form controls is shown in Fig. 2B. In a 
A
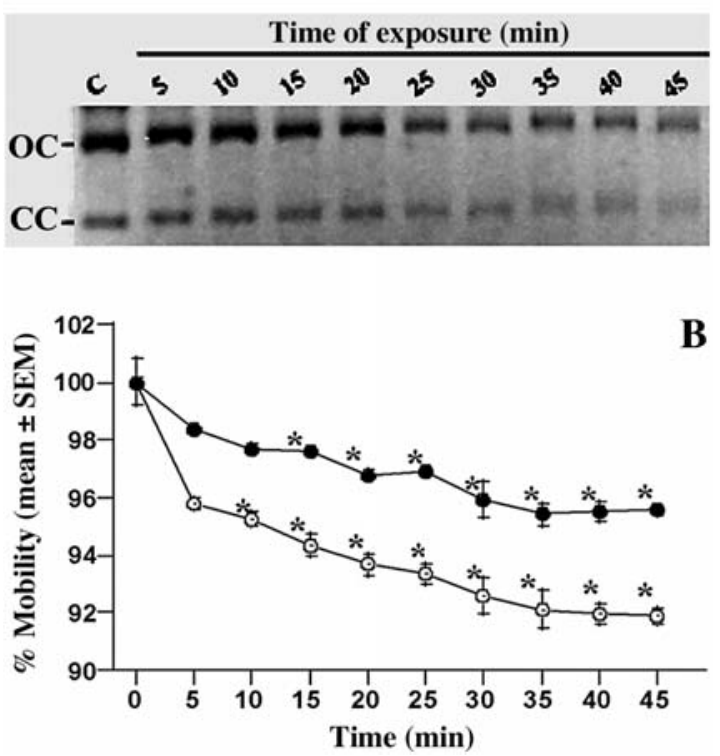

Figure 3. Effect of the duration of AEBN exposure on the gel electrophoretic mobility of plasmid pMTa4 in vitro. (A) Retardation of the mobility of the $\mathrm{CC}$ and $\mathrm{OC}$ forms of pMTa4 with increasing duration of exposure to AEBN [lane $1(\mathrm{C})$, unexposed pMTa4 $(4 \mu \mathrm{g})$; lanes 2-10, pMTa4 exposed to $250 \mu \mathrm{g}$ of AEBN for $5,10,15,20,25,30,35,40$ and 45 min respectively at $\left.37^{\circ} \mathrm{C}\right]$. (B) Percentage mobility changes for the $\mathrm{CC}(\bullet)$ and $\mathrm{OC}(\bullet)$ topological forms of pMTa4 as a function of the time of AEBN exposure. Data (mean \pm SEM) were obtained from the electropherogram (A). ${ }^{*}$ Statistically significant $(\mathrm{p} \leq 0.01)$ compared to the controls.

relative sense, the OC form of pMTa4 exhibited a higher retardation than the $\mathrm{CC}$ form.

AEBN exposure period-dependent gel mobility shift in pMTa4 DNA in vitro. The gel in Fig. 3A shows CC and OC topological forms of unexposed control pMTa4 isolate (lane 1) and pMTa4 exposed to $250 \mu \mathrm{g}$ of AEBN over increasing time periods (lanes 2-10). Retardation of the mobility of both the CC and $\mathrm{OC}$ forms is noticeable. The plot of percent mobility shift as compared to the mobility of the $\mathrm{CC}$ and $\mathrm{OC}$ form controls is shown in Fig. 3B. In a relative sense, the OC form of pMTa4 again exhibited a higher retardation than the $\mathrm{CC}$ form.

$A E B N$-induced increase in the molecular size of pMTa4. The gel in Fig. 4A shows NcoI linearized unexposed control pMTa4 (linear form or L; lane 1) and pMTa4 exposed to increasing doses of AEBN (lanes 2-6) for $30 \mathrm{~min}$ at $37^{\circ} \mathrm{C}$. An equivalent increase of $\sim 1000 \mathrm{bp}$ in the molecular size of the $\mathrm{L}$ form of pMTa4 was noticed at up to $150 \mu \mathrm{g}$ AEBN, after which it remained invariant (Fig. 4B).

Kinetics of the breakdown of adduct. Fig. 5A shows a gel of $\mathrm{CC}$ and $\mathrm{OC}$ topological forms of unexposed control pMTa4 (lane 1) and aqueous solutions of pMTa4 withdrawn every two hours from AEBN exposure at room temperature (lanes 2-13). The plot of percent mobility shift as compared to the mobility of the $\mathrm{CC}$ and $\mathrm{OC}$ form controls is shown in Fig. 5B. The graph shows that the maximum retardation in the mobility of the $\mathrm{CC}$ and $\mathrm{OC}$ forms of pMTa4 occurred between 6 and $8 \mathrm{~h}$. Subsequently, the trend progressively reversed. Both forms regained almost normal gel mobility by $24 \mathrm{~h}$.
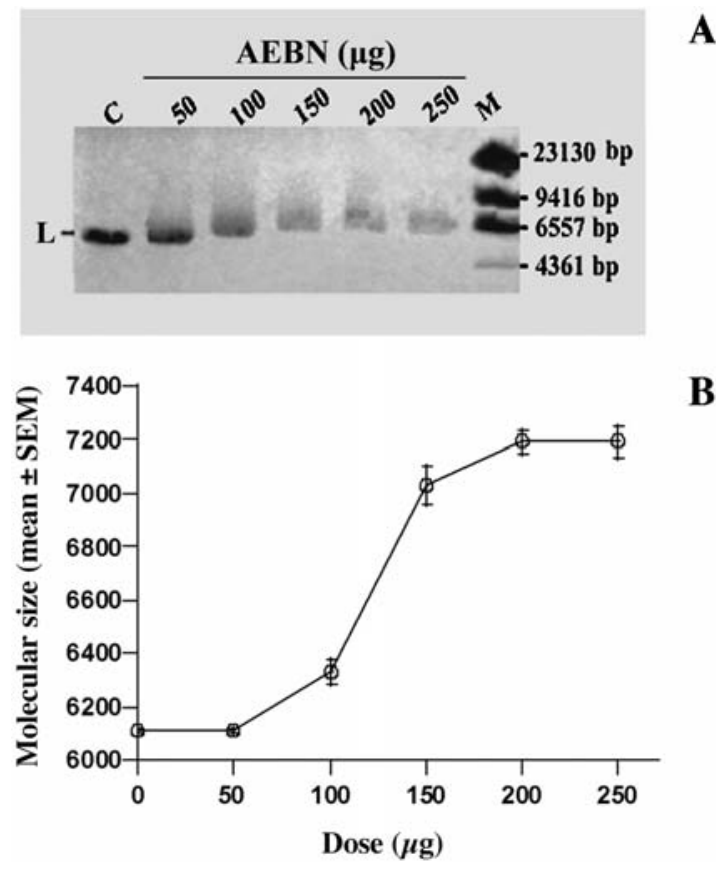

Figure 4. Increase in the molecular size of pMTa4 DNA upon exposure to AEBN. (A) Retardation of the mobility of linearized pMTa4 DNA exposed to increasing doses of AEBN [lane $1(\mathrm{C})$, unexposed pMTa4 $(4 \mu \mathrm{g})$; lanes 2-6, pMTa4 $(4 \mu \mathrm{g})$ exposed to 50, 100,150, 200 and $250 \mu \mathrm{g}$ AEBN respectively at $37^{\circ} \mathrm{C}$ for $30 \mathrm{~min}$; lane $7(\mathrm{M})$, DNA marker ( $\lambda$ DNA HindIII digest) in molecular size (bp)]. (B) Increase in bp of pMTa4 as a function of the dose of AEBN. Data (mean \pm SEM) were obtained from the electropherogram (A).

Effects of $\mathrm{pH}, \mathrm{Na}^{+}$and $\mathrm{K}^{+}$on the stability of adducts. The plots of percent mobility shift as compared to the mobility of the control CC and OC forms of pMTa4 under different treatment conditions are shown in Fig. 6. The results indicate that increasing $\mathrm{pH}$, in the range of $\mathrm{pH} 5.0-8.0$, did not essentially cause any change in the mobility of the $\mathrm{CC}$ and $\mathrm{OC}$ forms of pMTa4 (Fig. 6A). However, the presence of even trace amounts $(0.5 \mathrm{mmol})$ of either of the monovalent cations $\mathrm{K}^{+}$ (Fig. 6B) or $\mathrm{Na}^{+}$(Fig. 6C) slowed down the mobility of the $\mathrm{CC}$ and $\mathrm{OC}$ forms of pMTa4 as compared to the controls. The effect was more pronounced in the $\mathrm{OC}$ than the $\mathrm{CC}$ form.

\section{Discussion}

Adduct formation is known to cause a red shift in the absorption (21) and fluorescence (22) spectra of DNA in a dose-dependent manner. Our results (Fig. 1A) reveal that the spectrum of pMTa4 DNA shifted towards pure AEBN when the plasmid was exposed to $250 \mu \mathrm{g}$ of AEBN for $30 \mathrm{~min}$ at $37^{\circ} \mathrm{C}$. A significant $19.60 \mathrm{~nm}$ red shift of the spectrum of the mix suggests that AEBN interacted with pMTa4 DNA in vitro under the experimental conditions. Time as well as concentration kinetic studies were performed to determine the optimum or saturating dose and period of exposure to AEBN for such interaction. Fig. 1B shows an AEBN dose- and exposure time-dependent increase in absorption at $283.20 \mathrm{~nm}$. Since only one concentration of AEBN $(150 \mu \mathrm{g})$ per $4 \mu \mathrm{g}$ pMTa4 DNA at $37^{\circ} \mathrm{C}$ exhibited dose-dependence, we extrapolated that a concentration of AEBN above this would be saturating. Therefore, for all further in vitro studies, $4 \mu \mathrm{g}$ of pMTa4 was exposed to $250 \mu \mathrm{g}$ of AEBN for $30 \mathrm{~min}$ at $37^{\circ} \mathrm{C}$ 
$\mathbf{A}$
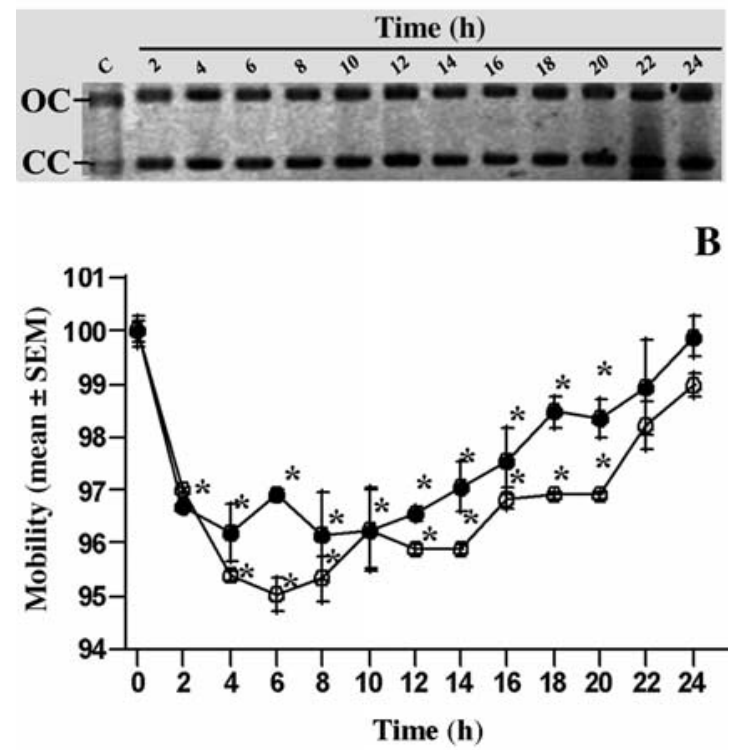

Figure 5. Time-dependent restoration of the mobility of pMTa4 upon the withdrawal of the AEBN exposure regime. (A) Change in the mobility of the $\mathrm{CC}(\bullet)$ and $\mathrm{OC}(\circ)$ topological forms of pMTa4 isolated from AEBN-exposed E. coli in vivo as a function of the time of AEBN exposure withdrawal [lane 1 (C), unexposed pMTa4; lanes 2-13, 2, 4, 6, 8, 10, 12, 14, 16, 18, 20, 22 and $24 \mathrm{~h}$ after the withdrawal of the AEBN exposure regime, respectively]. (B) Kinetics of the restoration of the mobility of the CC and OC forms of pMTa4 as a function of time after AEBN exposure withdrawal. Data (mean \pm SEM) were obtained from the electropherogram (A). *Statistically significant ( $\leq \leq 0.01)$ compared to the control.

in order to ensure that the maximum interaction of AEBN with pMTa4 occurred.

This interaction is likely to lead to adduct formation under experimental conditions (4). Our results show that the exposure of pMTa4 to AEBN caused statistically significant retardation of the mobility of both the $\mathrm{CC}$ and $\mathrm{OC}$ forms of the plasmid in an AEBN dose- (Fig. 2) and duration of exposure(Fig. 3) dependent manner. The OC form of the plasmid exhibited relatively higher retardation. Considering the fact that pMTa4 exposure to AEBN led to a red spectral shift, and that the mobility of the plasmid on agarose gel was retarded, one can conclude that the cause of both these observations was the formation of adducts on pMTa4 DNA upon its exposure to AEBN. The increased size of pMTa4 DNA due to adducts would reduce its mobility on agarose gel during electrophoresis. The nitrosation of arecoline has been shown to convert it into several species of electrophilic BSNA. This readily interacts with and forms adducts on DNA via weak interactions or chemical bonds, since the DNA is highly acidic (4). Arecoline or AEBN has also been shown to be mutagenic $(1,3,10)$. This strongly suggests that, in the experimental conditions used in the present study, AEBN-induced adducts were formed on pMTa4 DNA. The OC form of the plasmid exhibited a higher slope of curve of retardation of mobility than the CC form (Figs. 2B and 3B), suggesting that it had accumulated a higher quantum of adduct than the $\mathrm{CC}$ form. This is to be expected, as the OC form of plasmid is a relatively relaxed conformation (15-18), leading it to offer a higher probability of interaction with AEBN than the CC form under identical conditions. The chemical nature or identity of adducts has not been ascertained in this study. However, our observa-
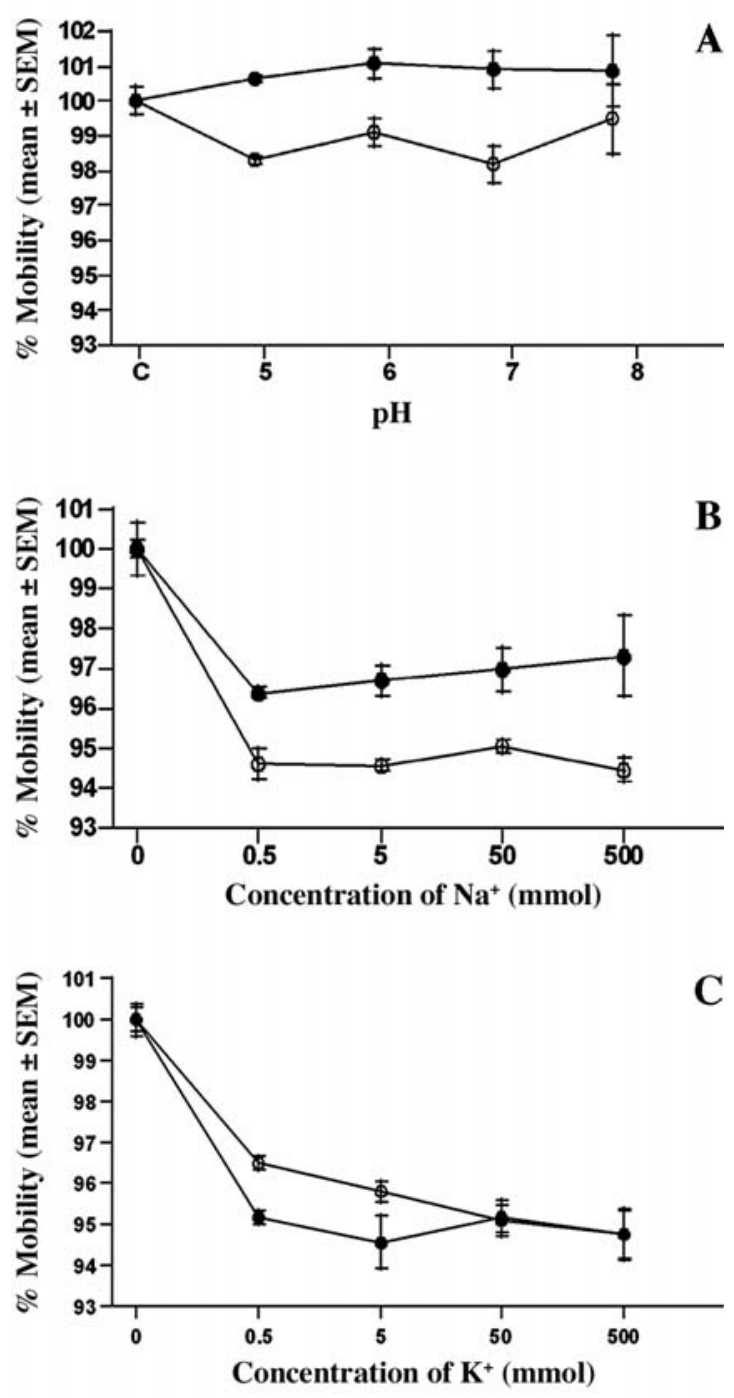

Figure 6. Effects of $\mathrm{pH}$ and the $\mathrm{Na}^{+}$and $\mathrm{K}^{+}$ions on the stability of AEBNinduced pMTa4 DNA adducts. (A-C) Mobilities of the CC (•) and OC ( $)$ topological forms of pMTa4 $24 \mathrm{~h}$ after incubation at different $\mathrm{pH}(\mathrm{A})$ or in the presence of increasing concentrations (mmol) of monovalent cations $\mathrm{Na}^{+}(\mathrm{B})$ and $\mathrm{K}^{+}(\mathrm{C})$. Controls were sham treated. Data (mean $\pm \mathrm{SEM}$ ) were obtained from the electropherograms. See text for further details.

tion of the formation of AEBN-induced DNA adducts (Figs. 2 and 3 ) is supported by reported biological observations of AEBN exposure resulting in DNA strand breaks (11), UDS (12), cell cycle alteration $(8,9,11)$, chromosomal aberrations (10), etc.

In order to determine the quantum of adducts formed on pMTa4 DNA in vitro by exposure to AEBN under saturating conditions, it was necessary to determine the precise change in the molecular size of pMTa4. As plasmid DNA is CC DNA, its migration on gel during electrophoresis is influenced by its net charge as well as its conformation. Consequently, the $\mathrm{CC}$ and OC forms of the plasmid, despite being identical in molecular size, show distinctly different migrations on gels (Figs. 2A, 3A and 4A). To quantify the increase in molecular size of pMTa4 DNA by AEBN exposure, it was therefore necessary for the plasmid to be initially linearized and then exposed to increasing doses of AEBN. The migration of the L form of plasmid DNA on gel during electrophoresis was considered to be directly proportional to its size. Fig. 4 shows 
that, with an increasing dose, the mobility of the $\mathrm{L}$ form of pMTa4 decreased progressively, reaching a maximum at $150 \mu \mathrm{g}$ of AEBN and remaining at that level for higher doses (Fig. 4B). The total increase in the molecular size of pMTa4 DNA was equivalent to $1000 \mathrm{bp}$ under the experimental conditions. The average molecular weights of nucleotides (NT) and BSNA are 283.25 and 126.6 Da, respectively. A shift of approximately $1000 \mathrm{bp}$ (corresponding to an increase of 2000 NT in two strands of DNA) was seen to occur in the L form of pMTa4 DNA. Hence, it follows that for a $1000 \mathrm{bp}$ increase in the molecular size, $\approx 1100$ adducts should have been formed on a piece of double-stranded DNA. Based on the results and the fact that pMTa4 DNA is $6173 \mathrm{bp}$ in size, it can be hypothesised that AEBN-induced adduct formation occurred every 3 NT under the experimental conditions.

To study the stability of the adducts on DNA, E. coli harboring pMTa4 was exposed to $2000 \mu \mathrm{g} / \mu \mathrm{l}$ of AEBN - a dose equivalent to the saturating dose of AEBN in vitro - under in vivo conditions in separate experiments. The isolated plasmid DNA was re-established in aqueous solution every $2 \mathrm{~h}$ for up to $24 \mathrm{~h}$, and then subjected to electrophoresis to determine the persisting extent of the retardation of mobility of the $\mathrm{OC}$ and $\mathrm{CC}$ forms. The result shows that retardation of mobility reached a maximum between 6 and 8 h (Fig. 5B; $6 \mathrm{~h}$ in the case of the $\mathrm{OC}$ form or $8 \mathrm{~h}$ in the case of the $\mathrm{CC}$ form). The trend then reversed and the $\mathrm{OC}$ and $\mathrm{CC}$ forms regained their normal mobilities in about $24 \mathrm{~h}$ (Fig. 5). As retardation in the mobility of a DNA band was due to adduct formation, progressive reversal indicates a loss of adducts from the DNA. This demonstrates that, after an aqueous solution of AEBNexposed pMTa4 was incubated for 6-8 h at room temperature, the adduct progressively began to dissociate from pMTa4 DNA. All adducts were lost by $24 \mathrm{~h}$, and the CC or OC forms of pMTa4 DNA, now devoid of any adduct, exhibited normal mobility on agarose gel. Therefore, the nature of AEBNinduced adducts on DNA appears to be unstable under the experimental conditions. There are many possible reasons for the observed loss of AEBN-induced adducts from pMTa4 DNA. The bonding between adduct and DNA could be noncovalent in nature, or could be based on weak interactions. Both such bonds could easily be broken. Alternately, as the $\mathrm{pH}$ of AEBN was acidic ( $\approx 5)$, adducts could undergo acid hydrolysis, or other natural components of $\mathrm{BN}$ included in the AEBN could contribute to the observed dissociation. This aspect shall be the subject of further investigation. However, in this study we conducted experiments to find the conditions which affect the observed instability of AEBN-induced adducts. $\mathrm{pH}$ in the range of 5.0-8.0 did not alter the instability of the adducts on either the CC or OC forms of pMTa4, as their mobility remained invariant (Fig. 6A). On the other hand, the presence of even trace amounts of monovalent cations conferred significant stability to the AEBN-induced pMTa4 DNA adducts (Fig. 6B and C). Due to biological relevance, two such cations, namely $\mathrm{Na}^{+}$and $\mathrm{K}^{+}$, were tested in this investigation. The results show that the presence of even trace amounts $(0.5 \mathrm{mmol})$ of $\mathrm{Na}^{+}$(Fig. $\left.6 \mathrm{~B}\right)$ or $\mathrm{K}^{+}($Fig. $6 \mathrm{C})$ conferred significant stability to the adducts. Since intracellular concentrations of $\mathrm{Na}^{+}$or $\mathrm{K}^{+}$in mammalian cells are in the range of 10 and $140 \mathrm{mmol}$ respectively (23), the results suggest that $\mathrm{BN}$-specific adducts on genomic DNA are likely to remain stable under normal metabolic conditions. The stability or continuity of adducts on genomic DNA may potentially create conditions for the induction and fixation of mutation, thereby strengthening the link between $\mathrm{BN}$ and the etiology of human carcinogenesis.

\section{Acknowledgements}

C.B. was financially supported by a doctoral research fellowship from the CSIR/UGC in the form of a JRF/SRF.

\section{References}

1. IARC: Tobacco habits other than smoking: betel quid and areca nut chewing and some related nitrosamines. In: Monograph on the Evaluation of the Carcinogenic Risk of Chemicals to Humans. Vol. 37. International Agency for Research on Cancer, Lyon, pp141-200, 1985.

2. IARC: Betel quid and areca nut chewing. In: Monograph on the Evaluation of the Carcinogenic Risk of Chemicals to Humans. Vol. 85. International Agency for Research on Cancer, Lyon, p39, 2004.

3. Sharan RN: Association of betel nut with carcinogenesis - a review. Cancer J 9: 13-19, 1996.

4. Chiba I: Prevention of betel quid chewer's oral cancer in the Asian-Pacific area. Asian Pac J Cancer Prev 2: 263-269, 2001.

5. Trivedy CR, Craig G and Warnakulasuriya S: The oral health consequences of chewing areca nut. Addict Biol 7: 115-125, 2002.

6. Péréz-Cabré M, Cervantes G, Moreno V, Prieto MJ, Pérez JM, Font-Bardia $\mathrm{M}$ and Solans X: Pd(II) and Pt(II) complexes with aromatic diamines: study of their interaction with DNA. J Inorg Chem 98: 510-521, 2004.

7. Gaskell M: Comparison of the mutagenic activity of the benzene metabolites, hydroquinone and para-benzoquinone in the supF forward mutation assay: a role for minor DNA adducts formed from hydroquinone in benzene mutagenicity. Mutat Res 554: 387-398, 2004.

8. Wary KK and Sharan RN: Cytotoxic and cytostatic effects of arecoline and sodium nitrite on human cells in vitro. Int J Cancer 47: 396-400, 1991.

9. Chang MC, Ho YS, Lee PH, Chan CP, Lee JJ, Hahn LJ, Wang YJ and Jeng JH: Areca nut extract and arecoline induced the cell cycle arrest but not apoptosis of cultured oral KB epithelial cells: association of glutathione, reactive oxygen species and mitochondrial membrane potential. Carcinogenesis 22: 1527-1535, 2001.

10. Kumpawat K, Deb S, Roy A and Chatterjee A: Genotoxic effects of raw betel-nut extract in relation to endogenous glutathione levels and its mechanism of action in mammalian cells. Mutat Res 538: 1-12, 2003.

11. Wary KK and Sharan RN: Aqueous extract of betel-nut of North-East India induces DNA strand breaks and enhances cell proliferation in vitro. J Cancer Res Clin Oncol 114: 579-582, 1988.

12. Sharan RN and Wary KK: Study of unscheduled DNA synthesis following exposure of human cells to arecoline and extracts of betel-nut in vitro. Mutat Res 278: 271-276, 1992.

13. Lin M-H, Wang C-J, Huang H-P, Chou M-Y and Chou F-P: The tumorigenic characteristics of lime-Piper betel quid-transformed JB6 cells. Arch Toxicol 78: 167-173, 2004.

14. Bartsch H, Rojas M, Nair U, Nair J and Alexandrov K: Genetic cancer susceptibility and DNA adducts: studies in smokers, tobacco chewers and coke oven workers. Cancer Detect Prev 23: 445-453, 1999.

15. Humtsoe JO, Schroeder $\mathrm{CH}$ and Sharan RN: Is there a relationship between nucleotide sequence and radiation induced DNA damage? In: Trends in Radiation and Cancer Biology. International Cooperation Bilateral Seminars series, vol. 29. Sharan RN (ed). Forschungszentrum Jülich GmbH, Jülich, pp29-32, 1998.

16. Odyuo MM and Sharan RN: Differential DNA strand breaking abilities of ${ }^{\circ} \mathrm{OH}$ and ROS generating radiomimetic chemicals and $\gamma$-rays: Study of plasmid, pMTa4, in vitro. Free Rad Res 39: 499-505, 2005.

17. Humtsoe JO, Schneeweiss FHA, Srivastava A, Sarma and Sharan RN: Biological effects induced by swift heavy ions of Lithium on aqueous solution of plasmid pMTa4. Radiat Effect Defect Solid 158: 603-607, 2003. 
18. Bhattacharjee $\mathrm{C}$ and Sharan RN: UV-C radiation induced conformational relaxation of pMTa4 DNA in Escherichia coli may be the cause of single strand breaks. Int J Radiat Biol 81: 919-927, 2005

19. Budiman ME, Alexander RW and Bierbach U: Unique base-step recognition by a platinum-acrodinylthiourea conjugate leads to a DNA damage profile complementary to that of the anticancer drug cisplatin. Biochem 43: 8560-8567, 2004.

20. Pariat T and Sharan RN: Qualitative changes in mice liver HMG proteins after low dose chronic administration of aqueous extract of betel nut and diethylnitrosamine. Hepatol Res 12: 177-185, 1988.
21. Geacintov NE, Cosman M, Mao B, Alfano A, Ibanez V and Harvey RG: Spectroscopic characteristics and site I/site II classification of cis and trans benzo(a)pyrene diolepoxide enantiomerguanosine adducts in oligonucleotides and polynucleotides. Carcinogen 12: 2099-2108, 1991.

22. Jiang G, Jankowiak R, Grubor N, Banasiewicz M, Small GJ, Skorvaga, M, van Houten B and States JC: Supercoiling DNA promotes formation of intercalated $c i s-\mathrm{N}_{2}$-deoxyguanine adduct and base-stacked trans-N2-deoxyguanine adduct by (+)-7R,8Sdihydrodiol-9S,10R-epoxy-7,8,9,10-tetra-hydrobenzo(a)pyrene. Chem Res Toxicol 17: 330-339, 2004.

23. Murray RK, Granner DK, Mayes PA and Rodwell VW. In: Harper's Illustrated Biochemistry, 26th edition. McGraw-Hill Professional, pp416, 2003. 